\title{
Lagrangian Multiplier and Riemannian Spaces
}

\author{
Cornelius Lanczos \\ Boeing Airplane Company, Seattle, Washington* \\ “. . . Wenn die Könige bauen, haben die Kärrner zu tun.”-Schiller**
}

\begin{abstract}
The Riemannian geometries, which are derivable from a quadratic action principle, are generated by a new mathematical approach, based on the method of the Lagrangian multiplier. This changes the 10 differential equations of fourth order for the $g_{i k}$ to 24 differential equations of second order for a new field quantity. This field quantity is a tensor of third order, antisymmetric in one pair of indices, in remarkable analogy to the fundamental $\Lambda$-tensor of Einstein's theory of distant parallelism. The field equations are tied together by 13 identities. The basic structure of the field equations for infinitesimal fields is investigated and their relations to Einstein's theory of gravity and Einstein's theory of distant parallelism discussed.
\end{abstract}

\section{INTRODUCTION}

$\mathrm{E}$ VER since Einstein's fundamental discovery [2]*** of the relation between Riemannian curved spaces and physics, the speculative mind was on the lookout for some geometrical clues which would shed some light on the nature of physical phenomena whose origin is not purely gravitational. The infinitesimal geometry of Weyl, the affine geometry of Eddington, Schouten, and recently Schroedinger, the five-dimensional theories of Kaluza, Einstein, Mayer, Bergmann, and others, the addition of "distant parallelism" to Riemannian geometry by Einstein, the projective relativity of Veblen and Hoffmann, and Pauli, are some of the major landmarks ${ }^{1}$ in the struggle for a natural generalization of the Einsteinian field equations, with the ultimate goal that eventually all physical action should be explicable as the natural emanation of a definite kind of space-time structure.

In recent years Einstein discovered a field theory based on "bivectors," 1 a and a field theory based on a Hermitian line-element. ${ }^{1 \mathrm{~b}}$

The author himself [7] followed a somewhat different path. Retaining the classical frame work of Riemannian geometry and adhering to the principle that the fundamental field equations shall be derivable from an action principle, he considered it plausible that the basic invariant of that principle should be quadratic rather than linear in the curvature components. ${ }^{2}$ In a later development [8], the theory of a statistically distributed carrier field of very high frequency was added. Such a metrical plateau can generate an average curvature

\footnotetext{
* Present address; Institute for Numerical Analysis, U.C.L.A., Los Angeles, Cal.

** "When kings are at work, the carpenters are kept busy."

*** References in [ ] are listed at the end of the article.

${ }^{1}$ It is impossible to enumerate all the original sources. The monography of Veblen [11] contains a representative bibliography (see p. 68) up to 1933, while the more recent literature is partially digested in the textbook of Bergmann [1]: see also Schroedinger [10] and subsequent articles.

1a A. Einstein and V. Bargmann, Ann. of Math. 45, 1, 15 (1944)

1b A. Einstein, Ann. of Math. 46, 578 (1945); A. Einstein and E. G. Strauss, Ann. of Math. 47, 731 (1946).

${ }^{2} \mathrm{H}$. Weyl [12] likewise advocated a quadratic action principle. However, in his infinitesimal geometry the fundamental quadratic invariant is much less uniquely determined; (see [12], p. 132).
}

radius of microscopic rather than astronomical dimensions, thus providing a universal length of sub-atomic order of magnitude for the construction of material particles.

One of the most serious objections to this line of thought is the fact that the basic field equations for the $g_{i k}$ come out as differential equations of fourth rather than second order. This makes any comparison with the classical field equations of mathematical physics extremely difficult since the fundamental field equations of nature seem to offer themselves as differential equations of first and second order. The present investigation completely eliminates this handicap by a new mathematical approach to the problem. Instead of operating with the $g_{i k}$, the components $R_{i k m n}$ of the Riemannian curvature tensor are considered as the basic metrical quantities, restricted by the Bianchi identities which are the auxiliary conditions of the variational problem. This gives rise to a Lagrangian multiplier which introduces a new fundamental field tensor. The fundamental field equations of the new formulation appear now as differential equations of only second order, of the same general structure as all the customary "nabla equations" of mathematical physics.

Of particular interest in this development is the fact that the new field tensor $H^{m}{ }_{i k}$ happens to be a tensor of third order, antisymmetric in $i$ and $k$. This brings the theory directly in close relation to Einstein's theory of "distant parallelism" which is likewise based on a tensor of third order, $\Lambda^{m}{ }_{i k}$, likewise antisymmetric in $i$ and $k$; (see [4], Eq. (10), p. 220). The principal difference between the two theories can be characterized as follows. In Einstein's theory the $\Lambda^{m}{ }_{i k}$ tensor is not a primitive field quantity but derived by differentiation from the fundamental ${ }_{s} h^{\nu}$. In the present theory the tensor $H^{m}{ }_{i k}$ is a primitive quantity, of the nature of a "generating function," which need not be reducible to a tensor of lower order, although for some important classes of solutions a reduction to a tensor of only second order actually occurs, as Sections VII and IX will demonstrate.

Another interesting fact deserves attention. The 
present theory is strictly shaped to the mathematical nature of a four-dimensional manifold. The Maxwellian equations of electromagnetic action, not less than Dirac's equation of the electron, strongly indicate that the specifically four-dimensional structure of the world is not accidental but deeply interwoven with the fundamental symmetry patterns of nature. The nabla operator is in both cases the result of a process which has no natural counterpart in manifolds of $n$ dimensions. Now in the theory pursued here, the nabla operation is not the result of a contraction, as in Einstein's theory, but of a specific transformation of Bianchi's identity which is possible only in four dimensions.

The present investigation is not devoted to any of the great unsolved problems of theoretical physics. It is strictly limited to infinitesimal fields and linear operators. Hence it cannot come to any valid conclusions concerning the nature of material particles. Yet even the first approximation reveals so many interesting relations to Einstein's own discoveries, that a brief outline of the theory may not be out of place at this illustrious occasion.

\section{A REFORMULATION OF BIANCHI'S IDENTITY IN FOUR DIMENSIONS}

The $2+2$ symmetry of a four-dimensional Riemannian world allows a remarkable re-interpretation of Bianchi's fundamental identity. Let us define the "dual" curvature tensor $R^{*}{ }_{i k m n}$ by the following operation:

$$
R^{*}{ }_{i k m n}=R^{\alpha \beta \mu \nu} \delta_{\alpha \beta i k} \delta_{\mu \nu m n},
$$

where $\delta_{\alpha \beta \mu \nu}$ denotes the completely antisymmetric "determinant tensor." Then the Bianchi identity for this new tensor appears as a regular divergence equation: ${ }^{3}$

$$
R^{* \alpha_{i k m, \alpha}}=0 \text {. }
$$

\section{THE METHOD OF THE LAGRANGIAN MULTIPLIER}

Let us now assume that our aim is to characterize a Riemannian manifold by an action principle which is quadratic in the curvature components. An investigation of the basic invariants available for this purpose shows [9] that only two independent invariants remain which may be combined by a constant factor. ${ }^{4} \mathrm{We}$ can choose

$$
R^{*}{ }_{i k m n} R^{* i k m n} \text { and } R^{2} \text {. }
$$

However, for infinitesimal fields the second invariant may be omitted. The terms resulting from this invariant are proportional to $R$. But the field equations which possess a quadratic action principle reveal the general peculiarity that $R=$ const. is an exact first integral of the field equations; (see [7], Eq. (5.4), p. 725). That

\footnotetext{
3 The Einsteinian sum convention is adopted throughout the paper. The "comma" refers to covariant differentiation.

${ }^{4}$ See also R. Weitzenboeck, Wien Ber. 129, 683 (1920); R. Bach, Math. Zeits. 9, 110 (1921); F. Juettner, Math. Ann. 87, 270 (1922).
}

constant must be practically zero to allow the existence of infinitesimal fields. But then the contribution of the second invariant is infinitesimal of the second order and thus negligible for our present purposes.

We do not want to express the $R_{i k m n}$ in terms of the $g_{i k}$ but consider them as basic metrical quantities, from which the $g_{i k}$, if the need arises, are uniquely obtainable (except for infinitesimal coordinate transformations), provided that the integrability conditions (II.2) are fulfilled. These conditions are thus the auxiliary conditions of our variation problem. They give rise to a Lagrangian multiplier $H^{m}{ }_{i k}$ in the form

$$
-\int H^{m}{ }_{i k, \alpha} R_{i k m \alpha} d \tau,
$$

( $d \tau=$ four-dimensional volume element) which has to be added to the action integral

$$
\frac{1}{2} \int\left(R_{i k m n}^{*}\right)^{2} d \tau
$$

For infinitesimal fields the covariant and contravariant components coincide, assuming that the basic Euclidean metric is normalized to the Pythagorean normal values

$$
g_{i k}=\delta_{i k} \text {. }
$$

Covariant differentiation can now' be replaced by ordinary differentiation.

In this investigation the upper and lower position of the indices shall not refer to covariant and contravariant components, since this distinction need not be made. The upper index in $H^{m}{ }_{i k}$ is chosen merely in order to set it apart from the two lower indices $i$ and $k$. The tensor $H^{m}{ }_{i k}$ is antisymmetric in $i$ and $k$, while the symmetry with respect to $m$ is not specified.

Performing the variation we obtain, in view of the algebraic symmetry properties of the Riemann tensor,

$$
R^{*}{ }_{i k m n}=H^{m}{ }_{i k, n}-H^{n}{ }_{i k, m}+H^{i}{ }_{m n, k}-H^{k}{ }_{m n_{\mathrm{r}} i} .
$$

This formula is structurally similar to the definition of $R_{i k m n}$ on the basis of the $\Gamma$-quantities. If we write this definition (for infinitesimal fields) in the symmetrized version

$$
R_{i k m n}=\frac{1}{2}\left[\Gamma_{m k, n}^{i}-\Gamma_{n k, m}^{i}+\Gamma^{m}{ }_{i n, k}-\Gamma_{k n, i}^{m}\right],
$$

we see that the tensor $H^{m}{ }_{i k}$ is analogous to $\Gamma_{m k}^{i}$. Yet the symmetry properties of these two tensors are quite different. The symmetry of $\Gamma$ exists with respect to $m$ and $k$, while the antisymmetry of $H$ exists with respect to $i$ and $k$. Moreover, $\Gamma^{i}{ }_{m k}$ is not a real tensor but can be made locally zero at any preassigned point of the manifold. On the other hand, $H^{m}{ }_{i k}$ is a real tensor of third order.

In the derivation of (III.5) we omitted the "cyclic identity" of the Riemannian curvature tensor which reduces the 21 algebraically independent components to 20. Specifically in four dimensions the cyclic identity 
may be written in form of the following scalar equation,

$$
R^{*}{ }_{i k m n} \delta^{i k m n}=0 \text {. }
$$

This leads to the following a priori restriction ${ }^{5}$ of the tensor $H^{m}$,

$$
H_{\alpha, \alpha}^{*}=0 \text {, }
$$

where $H^{*}{ }_{i}$ denotes the "dual vector" associated with the third-order tensor $H^{m}{ }_{i k}$,

$$
H^{*}{ }_{i}=H^{\mu}{ }_{\alpha \beta} \delta_{\alpha \beta \mu i} \text {. }
$$

The Eq. (III.8) expresses the "conservation law" for the vector $H^{*}$.

\section{THE BASIC FIELD EQUATIONS}

If the explicit expression (III.5) is substituted into the Bianchi identity (II.2), the following fundamental determining equation is obtained for the tensor $H^{m}{ }_{2 k}$ (using the notation $\Delta$ for the Laplacian operator $\left.\partial^{2} / \partial x_{\alpha}^{2}\right)$,

$$
P^{m}{ }_{i k} \equiv \Delta H^{m}{ }_{i k}-H_{i k, m}+H^{m}{ }_{i, k}-H^{m}{ }_{k, i}=0,
$$

where two independent tensors of second order $H_{i k}$ and $H^{m}{ }_{i}$ are introduced on the basis of the divergence operations,

and

$$
H_{i k}=H^{\alpha}{ }_{i k, \alpha},
$$

$$
H^{m}{ }_{i}=H^{i}{ }_{m \alpha, \alpha} .
$$

We thus obtain a system of 24 partial differential equations of second order for the determination of the 24 field quantities $H^{m}{ }_{i k}$.

\section{THE BASIC IDENTITIES}

The decisive importance of identities in any generally covariant system of field equations was often emphasized by Einstein. One of the strongest assets of the contracted curvature tensor $R_{i k}-\frac{1}{2} R g_{i k}$ is the fact that its divergence vanishes identically. This gives rise to the conservation laws of momentum and energy, and leads to the geodetic motion law of general relativity. In the theory of distant parallelism Einstein used the principle of identities as a selection principle for setting up the basic field equations [6], obtaining a system of $16+6=22$ field equations with 10 identities for the determination of the $16{ }_{s} h^{\nu}$ quantities, 4 of which can be normalized due to the free choice of the coordinates. ${ }^{6}$

In the present theory two independent divergence identities can be derived, according to whether we differentiate with respect to the upper or one of the lower indices. First we obtain

$$
P^{\alpha}{ }_{i k, \alpha} \equiv 0 \text {. }
$$

${ }^{5}$ In principle the Eq. (III.7) gives rise to an additional scalar Lagrangian factor. One can show, however, that this factor may be omitted and replaced by the condition (III.8), without loss of generality.

${ }^{6}$ See also the author's report, Die neue Feldtheorie Einsteins, in Ergeb. d. exakt. Naturwiss. 10, 97 (1931).
Moreover

$$
P_{i \alpha, \alpha}^{k} \equiv \Delta\left(H_{i}^{k}+H_{k}^{i}\right)-\left(\varphi_{i, k}+\varphi_{k, i}\right),
$$

where we have put

$$
\varphi_{i}=H_{i \alpha, \alpha}=H^{\alpha}{ }_{i, \alpha} .
$$

Note that the right side of (V.2) is symmetric with respect to $i$ and $k$. This gives rise to the identity

$$
\left(P_{i \alpha}^{k}-P_{k \alpha}^{i}\right)_{, \alpha} \equiv 0 \text {. }
$$

The Eqs. (V.1) and (V.4) represent 12 divergence identities, thus reducing the number of independent field equations to $24-12=12$, in strange coincidence to the $16-4=12$ independent field equations which are demanded in Einstein's theory of distant parallelism.

One additional divergence identity can be established in view of the $a$ priori restriction (III.8). We obtain

where

$$
P_{\alpha, \alpha}^{*} \equiv 0,
$$

$$
P_{i}^{*}=P^{\mu}{ }_{\alpha \beta} \delta_{\alpha \beta \mu i} .
$$

\section{NORMALIZATION OF THE TENSOR $H_{i k}$}

The expression (III.5) shows that the addition to $H^{m}{ }_{i k}$ of the gradient

$$
G_{i k, m}
$$

has no influence on $R^{*}{ }_{i k m n}$. Hence the field equations are unable to determine $H^{m}{ }_{i k}$ closer than the gradient of an arbitrary antisymmetric tensor of second order. The freedom of adding a gradient of the form (VI.1) may be used to normalize some property of $H^{m_{i k}}$, eliminating at the same time the uncertainty in the solution of the field equations. The most natural normalization is obtained if we make $H_{i k}$ equal to zero;

Then also

$$
H_{i k}=H^{\alpha}{ }_{i k, \alpha}=0 \text {. }
$$

$$
\varphi_{i}=0 \text {. }
$$

The normalization (VI.2) corresponds to the equation

$$
\Lambda^{\alpha}{ }_{i k, \alpha}=0
$$

adopted by Einstein in his theory of distant parallelism; (see [6], Eq. (12), p. 21). Moreover, the vanishing of the vector (VI.3) corresponds to the normalization of infinitesimal gravitational fields of the form

$$
g_{i k}=\delta_{i k}+\gamma_{i k}
$$

by the Einsteinian condition

$$
\left(\gamma_{i \alpha}-\frac{1}{2} \gamma \delta_{i \alpha}\right)_{, \alpha}=0 .
$$

With this normalization the Einsteinian field equations of gravity for infinitesimal fields separate into the pure nabla equations,

$$
\Delta \gamma_{i k}=0 \text {. }
$$

The normalization (VI.2) has similar effects for the theory here discussed, as Section $\mathrm{X}$ will show. 
VII. SOLUTION OF THE FIELD EQUATIONS BY A SYMMETRIC TENSOR OF SECOND ORDER

The general structure of the field equations suggests a solution of the specific character,

$$
H^{m}{ }_{i k}=F_{m i, k}-F_{m k, i} \text {. }
$$

Substitution into (III.5) shows that the antisymmetric part of the tensor $F_{i k}$ has no influence on $R^{*}{ }_{i k m n}$. Hence we can assume that $F_{i k}$ is symmetric in $i$ and $k$;

$$
F_{i k}=F_{k i} \text {. }
$$

Although the reduction of a tensor of third order to a symmetric tensor of second order strongly overdetermines the field equations, yet a solution is possible under these circumstances. The normalization (VI.2) demands the condition

$$
F_{i \alpha, \alpha}=\psi_{, i}
$$

where $\psi$ is an arbitrary scalar function. The field equations now give

$$
P^{m}{ }_{i k} \equiv 2 \Delta F_{m i, k}-2 \Delta F_{m k, i}=0 .
$$

These equations lead to the nabla equations for $F_{i k}$,

$$
\Delta F_{i k}=0 .
$$

We note the complete analogy which exists between the Eqs. (VII.5), supplemented by the auxiliary condition (VII.3), and the Einsteinian field Eqs. (VI.7) and (VI.6) for infinitesimal gravitational fields. If we choose

and

$$
F_{i k}=\gamma_{i k},
$$

$$
\psi=\frac{1}{2} \gamma,
$$

then the two systems of field equations coincide. We thus see that any Einstein field provides a particular solution of the field Eqs. (IV.1).

This correlation is by no means trivial. It establishes the interesting fact that for any given Einstein field a second Einstein field can be constructed, defined by the correlation

$$
\bar{R}^{*}{ }_{i k m n}=R_{i k m n} \text {. }
$$

In view of the strongly overdetermined nature of the Riemannian curvature tensor, such a correlation cannot be expected, except under strongly restricted conditions. Yet the only condition demanded is the characteristic property of the Einstein fields, viz. the vanishing of the matter tensor.

\section{THE MATTER TENSOR $-\boldsymbol{R}^{*}{ }_{i k}$}

If in (III.5) we contract over $i$ and $m$ and change the sign, we obtain the matter tensor of general relativity,

$-R^{*}{ }_{i k} \equiv R_{i k}-\frac{1}{2} R \delta_{i k}=\phi_{i, k}+\phi_{k, i}-\left(H^{i}{ }_{k}+H^{k}{ }_{i}\right)$.

The vector $\phi_{i}$ is here defined as

$$
\phi_{i}=H_{i \alpha}^{\alpha} \text {. }
$$

In the case of the particular solution considered in the previous chapter, we obtain

$$
\begin{aligned}
\phi_{i} & =(\psi-F)_{, i}, \\
H^{k} & =H^{i}{ }_{k}=-\psi, i k
\end{aligned}
$$

(with the notation $F=F_{\alpha \alpha}$ ). This gives

$$
-R_{i k}^{*}=2(2 \psi-F)_{i k} \text {. }
$$

In particular, the choice (VII.6), (VII.7) yields

$$
R^{*}{ }_{i k}=0 \text {. }
$$

Generally, however, the relation $2 \psi=F$ is not demanded and we obtain a class of geometries which is slightly more general than the Einsteinian class. ${ }^{7}$

\section{SOLUTION OF THE FIELD EQUATIONS BY A NON-SYMMETRIC TENSOR OF SECOND ORDER}

A second analogous class of geometries is of particular interest because of its close relation to the field equations advocated by Einstein in his theory of distant parallelism. In this class of solutions the symmetry of $F_{i k}$ is no longer required.

Once more we follow the course of Section VII, but with one essential difference. The antisymmetric index pair $i k$ always possesses a "dual" index pair (e.g., the dual of 12 is 34 , the dual of 13 is 42 , etc.). Let us denote the dual pair of $i \dot{k}$ by $\underline{i k}$. Then we can define the dual of the tensor $H^{m}{ }_{i k}$ with respect to the antisymmetric index pair $i k$ by $H^{m_{i k}}$. It is now this dual tensor $H^{m_{i k}}$ that we want to reduce in the previous fashion to a tensor of second order, $F_{i k}$;

$$
H^{m_{i k}}=F_{m i, k}-F_{m k, i} \text {. }
$$

This relation imitates the dependence of Einstein's $\Lambda^{m}{ }_{i k}$ tensor on the $h_{m \alpha}$ for infinitesimal fields. In contrast to the case treated in Section VII, the symmetry of $F_{m i}$ is not demanded; hence we have 16 tensor components at our disposal, in analogy to the 16 components of the four vectors $h_{m \alpha}$. We wish to investigate what the field equations request of this class of solutions and how the results compare with the results deduced from Einstein's field equations.

If we evaluate the tensor (IV.3) for our solution, we find that

$$
H^{i}{ }_{k}=0 .
$$

Moreover, the normalization (VI.2) yields

$$
F_{\alpha i, \alpha}=0 \text {, }
$$

while the field equations demand

$$
\Delta F_{i k}=0 .
$$

Let us now split $F_{i k}$ into a symmetric and an antisymmetric part,

$$
F_{i k}=S_{i k}+\sigma_{i k},
$$

7 The generalization may be characterized as follows. The Einsteinian $d s^{2}$ is multiplied by the factor $1+\epsilon p$ where $\epsilon$ is small, while $p$ is an arbitrary potential function $(\Delta p=0)$. 
where $S_{i k}$ is symmetric and $\sigma_{i k}$ antisymmetric. More- gives over, let us put

$$
\begin{aligned}
S_{i \alpha, \alpha} & =u_{i}, \\
\sigma^{*}{ }_{i \alpha, \alpha} & =v_{i} .
\end{aligned}
$$

According to (VIII.1) we obtain

where

$$
-R^{*}{ }_{i k}=\phi_{i, k}+\phi_{k, i}
$$

$$
\phi_{i}=2 v_{i}
$$

The four quantities $v_{i}$ are the only ones by which the antisymmetric tensor $\sigma_{i k}$ participates in the curvature components. Hence we can discard the $\sigma_{i k}$ themselves and operate with the vector $v_{i}$ alone. This vector satisfies the nabla equation

$$
\Delta v_{i}=0
$$

and also the divergence equation

$$
v_{\alpha, \alpha}=0 \text {. }
$$

Hence $\phi_{i}$ has all the earmarks of the "vector potential" of electromagnetism.

The Eq. (IX.8) appeared in one of Einstein's early papers on distant parallelism (see [5], Eq. (7), p. 226), together with the tentative assumption that $\phi_{i}$ may represent the electromagnetic vector potential. In the later phases of the theory Einstein dropped that interpretation, in favor of declaring the antisymmetric part of the $h_{m \nu}$ as the electromagnetic field strength. ${ }^{8}$

The vector $u_{i}$ likewise satisfies the nabla equations and the conservation law

$$
\begin{aligned}
\Delta u_{i} & =0, \\
u_{\alpha, \alpha} & =0 .
\end{aligned}
$$

In addition, however, the two vectors $u_{i}$ and $v_{i}$ are not independent of each other but tied together by the following relation:

$$
v_{i, k}-v_{k, i}+\left(u_{i, k}-u_{k, i}\right)^{*}=0 .
$$

The conditions (IX.12) and (IX.13) guarantee the solvability of these equations. They determine the $v_{i}$ uniquely, short of the gradient of a scalar potential function.

Let us first assume that $F_{i k}$ is symmetric. Then

and we must have

$$
v_{i}=0 \text {, }
$$

$$
u_{i}=S_{i \alpha, \alpha}=\psi, i,
$$

which imitates the relation (VII.3). In this case (IX.9)

${ }^{8}$ The symmetry structure of the theory discussed here is such that there are several possibilities open for an interpretation of the Maxwellian equations. However, an investigation of purely infinitesimal fields cannot come to any binding conclusions as to the translation of a field theory into physical reality, because the physical manifestation of the field equations demands the derivaation of a definite law of motion for the particle. This, however, is not possible without the investigation of the second order interaction effects between fields, caused by the non-linearity of the field equations.

$$
R^{*}{ }_{i k}=0 \text {, }
$$

and the field is free of matter. Once more we can identify $S_{i k}$,with the components $\gamma_{i k}$ of an infinitesimal gravitational field (see Eqs. (VI.5)-(VI.7)) and once more we come to an interesting correspondence property of purely gravitational fields, in the sense that an arbitrary Einstein field can serve for the generation of another Einstein field. The correlation is now

$$
\bar{R}_{i k m n}^{*}=R_{i k m n}+R_{\underline{i k m n},}
$$

which is different from the correlation (VII.8), obtained in the previous class of solutions.

We now come to the discussion of the general case in which $v_{i}$ is not zero. We do not get that independence of "gravitational" and "electromagnetic" fields that Einstein found on the basis of his field equations; (see [6], Eqs. (19), (20), p. 23). The vector $v_{i}$ cannot exist without the vector $u_{i}$. It is the violation of the divergence condition for $S_{i k}$. which causes the "electromagnetic" part of the field. While a purely gravitational field can exist without electromagnetism, yet "pure electromagnetism" is not possible. The electromagnetic part of the field (if this interpretation is permissible) requires "gravitational support" for its existence, since it is determined by the vector $u_{i}$ (see Eq. (IX.6)) which is generated by the symmetric tensor $S_{i k}$.

\section{GENERAL SOLUTION OF THE INFINITESIMAL FIELD EQUATIONS}

If our desire is to get away from particular solutions and obtain the complete class of possible solutions, we have to include the entire space-time manifold in our considerations. On the other hand, the conditions for infinitesimal fields are realized in restricted portions of the space-time world only. In the central part of material particles the fields become too strong for infinitesimal considerations. And yet, it is still possible to include these fields in our discussions if we agree that we "round off" the sharp increases of the field functions, replacing them by fictitious values which remain infinitesimal everywhere. By this procedurewhich may be illustrated by replacing the Laplacian equation with singularities by Poisson's equation without singularities-we will not come to any valid conclusions concerning the non-linear inner portions of the field, but we will come to valid conclusions concerning the outer linear portions of the field. ${ }^{9}$

This method achieves the "linearization" of a set of non-linear differential equations by putting something on the right side of the equations. Hence a non-linear homogeneous set of equations is replaced by a linear

${ }^{9}$ This method of "flattening out" strong central fields, at the cost of a fictitious "right side" of the field equations which, however, must satisfy the conservation laws, was utilized by the author for the derivation of the motion law of general relativity for the center of mass of a particle (see Zeits. f. Physik 59, 514 (1930)). 
but inhomogeneous set of equations, which in our of the tensor $F_{i \kappa}$, case may be written as

$$
P^{m}{ }_{i k}=\kappa \rho^{m}{ }_{i k},
$$

where $\kappa$ is a small constant. These equations correspond to the inhomogeneous Einsteinian gravitational equations for infinitesimal fields,

$$
R_{i k}-\frac{1}{2} R g_{i k}=\kappa \rho_{i k} .
$$

The right side $\rho^{m}{ }_{i k}$ is different from zero only in the non-linear central portions of the field. It is strongly restricted by the demand for "eigen solutions." These restrictions are not at our disposal. But another set of restrictions are at our disposal, viz. the a priori restrictions demanded by the identities of Section V. They yield the conditions

$$
\begin{aligned}
\rho^{\alpha}{ }_{i k, \alpha} & =0, \\
\left(\rho_{i \alpha}^{k}-\rho_{k \alpha}^{i}\right)_{, \alpha} & =0, \\
\rho_{\alpha, \alpha}^{*} & =0 .
\end{aligned}
$$

In comparison, the Einsteinian equations (X.2) demand only one vectorial condition

$$
\rho_{i \alpha, \alpha}=0 .
$$

Moreover, the tensor $\rho^{m}{ }_{i k}$ has to be prescribed as antisymmetric in $i$ and $k$, while the Einsteinian $\rho_{i k}$ is symmetric in $i$ and $k$.

The solution of the Einsteinian equations (X2) separates into the 10 nabla equations

$$
\Delta\left(\gamma_{i k}-\frac{1}{2} \gamma \delta_{i k}\right)=\kappa \rho_{i k}
$$

under the Einsteinian normalization condition

$$
\left(\gamma_{i \alpha}-\frac{1}{2} \gamma \delta_{i \alpha}\right)_{, a}=0
$$

which is guaranteed because of Eq. (X.6) (see [-3], Eqs. (3)-(5), pp. 689, 690).

A similar solution can be established for the field Eqs. (X.1). Let us put

$$
H^{m_{i k}}=\bar{H}^{m_{i k}}+F_{m i, k}-F_{m k, i},
$$

where the tensor $\bar{H}^{m}{ }_{i k}$ shall be defined by the 24 separated nabla equations,

$$
\Delta \bar{H}^{m}{ }_{i k}=\kappa \rho^{m}{ }_{i k},
$$

while the symmetric tensor $F_{m i}$ shall be defined as

$$
\Delta F_{i k}=-\frac{1}{2} \bar{H}^{k}{ }_{i}
$$

The symmetry of $\bar{H}_{i}^{k}=\bar{H}^{i}{ }_{k}$ follows from (X.4). Moreover, the condition (X.3) gives

$$
\bar{H}_{i k}=0 \text {. }
$$

Consequently $\bar{H}_{i}{ }_{i}$ is restricted by the divergence condition

$$
\bar{H}^{\alpha}{ }_{i, \alpha}=0,
$$

and that again guarantees the normalization condition

$$
F_{i \alpha, \alpha}=0 \text {. }
$$

Substitution of (X.9) into the field Eqs. (X.1) shows that the field equations are actually satisfied.

\section{QUADRATIC REPRESENTATIONS OF THE BASIC FIELD TENSOR}

It seems plausible to try solutions of the field equations for the following type of generating tensor

$$
H^{m}{ }_{i k}=F_{i k} \phi_{m},
$$

where $F_{i k}$ is an antisymmetric tensor of second order, while $\phi_{m}$ is a vector. Another class of representations has the form,

$$
H^{m}{ }_{i k}=F_{m i} \phi_{\kappa}-F_{m \kappa} \phi_{i},
$$

where the symmetry structure of $F_{m i}$ is not specified. Such solutions cannot be studied on the basis of purely linear operators. They demand the knowledge of the field in first and second approximation. Hence they are outside the limits of the present investigation which was solely devoted to the general exploration of infinitesimal fields.

\section{ACKNOWLEDGMENTS}

The basic idea of the present investigation occurred to the author during a lecture course in Relativity, given at the University of Washington during the fall quarter of 1948, at the invitation of the Physics Department. The author gratefully acknowledges the many stimulating discussions with his students; he is also greatly indebted to the administration of the Boeing Airplane Company, in particular to Dr. C. K. Stedman, Head of the Physical Research Unit, for the generous support and encouragement extended to him in all his theoretical endeavors.

\section{BIBLIOGRAPHY}

1. P. G. Bergmann, Introduction to the Theory of Relativity (Prentice-Hall, Inc., New York, 1942).

2. A. Einstein, "Die Grundlagen der Allgemeinen Relativitätstheorie," Ann. d. Physik 49, 769 (1916).

3. A. Einstein, Naeherungsweise Integration der Feldgleichungen der Gravitation (Berliner Berichte, 1916), p. 689.

4. A. Einstein, Riemann-Geometrie mit Aufrechterhaltung des Begriffs des Fernparallelismus (Berliner Berichte, 1928), p. 217.

5. A. Einstein, Neue Moeglichkeit fuer eine einheitliche Feldtheorie von Gravitation und Elektrizitaet (Berliner Berichte, 1928), p. 224.

6. A. Einstein, Die Kompatibilitaet der Feldgleichungen in der einheitlichen Feldtheorie (Berliner Berichte, 1930), p. 18.

7. C. Lanczos, Phys. Rev. 39, 716 (1932).

8. C. Lanczos, Phys. Rev. 61, 713 (1942).

9. C. Lanczos, Ann. of Math. 39, 842 (1938).

10. E. Schroedinger, Proc. Roy. I.A. A49, 43 (1943).

11. O. Veblen, Projektive Relativitaetstheorie (Verlag Julius Springer, Berlin, 1933).

12. H. Weyl, "Eine neue Erweiterung der Relativitaetstheorie," Ann. d. Physik 59, 101 (1919). 